\title{
Test-beam studies of diamond sensors for SLHC
}

\author{
${ }^{\mathrm{a}}$ Lorenzo Uplegger*, ${ }^{\mathrm{b}}$ Jennifer Ngadiuba, ${ }^{\mathrm{c}}$ Enver Alagoz, ${ }^{\mathrm{a}} \mathrm{Jeff}$ Andresen, ${ }^{\mathrm{c}}$ Kirk Arndt, ${ }^{\mathrm{c}}$ Gino \\ Bolla, ${ }^{\mathrm{C}}$ Daniela Bortoletto, ${ }^{\mathrm{d}} \mathrm{Jean}$ Marie Brom, ${ }^{\mathrm{e}} \mathrm{Rich}$ ard Brosius, ${ }^{\mathrm{C}}$ Mayur Bubna, ${ }^{\mathrm{a}} \mathrm{John}$ \\ Chramowicz, ${ }^{\mathrm{f}}$ John Cumalat, ${ }_{\mathrm{f}}^{\mathrm{F}}$ rank Jensen, ${ }^{\mathrm{c}}$ Alex Krzywda, ${ }^{\mathrm{e}}$ Ashish Kumar, ${ }^{\mathrm{a}}$ Simon Kwan, \\ ${ }^{\mathrm{a}} \mathrm{CM}$ Lei, ${ }^{\mathrm{b}}$ Dario Menasce, ${ }^{\mathrm{b}}$ Luigi Moroni, ${ }^{\mathrm{g}}$ Margherita Obertino, ${ }^{\mathrm{h}}$ Ilya Osipenkov, ${ }^{\mathrm{i}}$ Lalith \\ Perera, ${ }^{\mathrm{a}}$ Alan Prosser, ${ }^{\mathrm{a}}$ Ryan Rivera, ${ }^{\mathrm{g}}$ Ada Solano, ${ }^{\mathrm{a}}$ Ping Tan, ${ }^{\mathrm{b}}$ Stefano Terzo, ${ }^{\mathrm{a}}$ Nhan Tran, \\ ${ }^{\mathrm{f}}$ Stephen Robert Wagner
}

a. Fermi National Laboratory

b. Istituto Nazionale di Fisica Nucleare, Sezione di Milano Bicocca

c. Purdue University

d. Institut Pluridisciplinaire Hubert Curien Strasbourg

e. University of Buffalo

f. University of Colorado Boulder

g. Istituto Nazionale di Fisica Nucleare, Sezione di Torino

h. Texas A\&M

i. $\quad$ Mississippi University

\begin{abstract}
:
Diamond sensors are studied as an alternative to silicon sensors to withstand the high radiation doses that are expected in future upgrades of the pixel detectors for the SLHC. Diamond pixel sensors are intrinsically radiation hard and are considered as a possible solution for the innermost tracker layers close to the interaction point where current silicon sensors cannot cope with the harsh radiation environment. An effort to study possible candidates for the upgrades is undergoing using the Fermilab test-beam facility, FTBF, where diamonds and 3D silicon sensors have been studied. Using a CMS pixel-based telescope built and installed at the FTBF, we are studying charge collection efficiencies for un-irradiated and irradiated devices bump-bonded to the CMS PSI46 pixel readout chip. A description of the test-beam effort and preliminary results on diamond sensors will be presented.
\end{abstract}

Key words: Tracking detectors, Diamond detectors, Solid state detectors, Test-beam

\section{Introduction*}

2

3 The innermost tracking device of the CMS general purpose 4 detector [1] at the Large Hadron Collider (LHC) at CERN, 5 consists of three Barrel layers and four Forward disks whose 6 basic building blocks are highly segmented silicon sensors 7 (pixels) tightly coupled with their corresponding PSI46V2 [2] 8 readout chips (ROCs). The high radiation doses that these 9 devices will have to withstand after the future LHC upgrades 10 is so high that the currently installed planar silicon sensors 11 will be damaged too quickly to be a practical solution. In order 12 to increase the radiation hardness of pixel detectors different 13 strategies are being pursued and test-beam around the world 14 are conducted to make sure that the performances of these 15 sensors, after a heavy irradiation comparable to the doses that 16 are envisioned at the SLHC, can still guarantee tracking 17 capabilities to meet the physics goals. An effort to study 18 possible candidates for the upgrades is undergoing at the 19 Fermilab test-beam facility, FTBF, where diamonds and 3D 20 silicon sensors are being studied. In this paper we'll focus our 21 attention to the description of the test-beam architecture and to 22 some preliminary results on diamond sensors.

* This work was supported by Fermi National Accelerator Laboratory operated by Fermi Research Alliance, LLC under Contract No. DEAC02-07CH11359 with the United States Department of Energy. L. Uplegger is with Fermi National Accelerator Laboratory, Batavia, IL 60510 USA (e-mail: uplegger@fnal.gov).

\section{2. Experimental setup}

27

28 The beam-tests were performed in 2012 at the FTBF with a $29120 \mathrm{GeV} / \mathrm{c}$ protons beam incident on an 8 planes pixel30 detector telescope. This telescope consists of 8 modules 31 leftover from the CMS Forward pixel detector production [2]. 32 They are based on the PSI46v2 Read Out Chip (ROC) [3] , 33 with $150 \mu \mathrm{m} \times 100 \mu \mathrm{m}$ pixel size, arranged in two sections, 34 with two Detectors Under Test (DUTs) placed between the 35 two telescope stations. Enhanced resolution is derived from 36 charge sharing by tilting the telescope planes at $25^{\circ}$. The 37 telescope resolution on the DUTs is as small as $6 \mu \mathrm{m}$ both in $38 \mathrm{X}(150 \mu \mathrm{m})$ and $\mathrm{Y}(100 \mu \mathrm{m})$ coordinates.

39 The Data Acquisition (DAQ) hardware is based on the 40 CAPTAN system [4] , developed at Fermilab, which uses a 41 gigabit Ethernet link to transfer the data from the experimental 42 area to the DAQ computer where data are stored. The 43 software, also developed at Fermilab, runs on a Windows PC 44 and is a suite of multithreaded applications written in C++. A 45 Data Quality Monitor runs in parallel, while data are acquired 46 to provide immediate feedback to spot any problem in real 47 time.

48

493. Offline analysis

50

51 In order to achieve the necessary resolution on the DUTs to 52 allow for detailed studies on resolution and charge sharing, 53 careful attention needs to be given to the alignment of the 54 telescope and DUTs detectors in the beam. A dedicated 55 software, called Monicelli, has been developed at the 56 University of Milano Bicocca which achieves a track 
1 resolution on the DUTs as small as $6 \mu \mathrm{m}$. Tracks are saved in 2 ROOT format to be later analyzed by another program, called 3 Chewie; the results of that analysis are discussed below.

4 We'll focus our attention on the preliminary results of 5 efficiency and charge collection for two diamond detectors, 6 both $500 \mu \mathrm{m}$ thick but with different crystal structure: mono 7 and poly crystalline.

\section{8}

\section{0}

\section{Charge collection}

11 The charge collected in the two detectors varies greatly due 12 to the intrinsic nature of the two crystalline structures. The 13 mono-crystal has a charge collection distance (CCD) greater 14 than $500 \mu \mathrm{m}$ and the charge collected is thus around $20000 e$ 15 while the poly-crystal with its $172 \mu \mathrm{m}$ CCD only collects 16 about $3400 e^{-}$as the Landau charge profiles show in Figure 1.

17
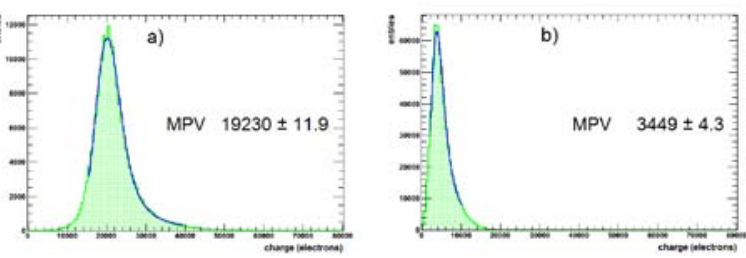

18

Figure 1: a) Charge collected in the mono-crystal with most probable value 20 (MPV) around $20000 e^{-}$while b) is the charge collected in the poly-crystal with 21 MPV value around $3400 e^{-}$.

22

23 In Figure 2 instead we show the charge distribution in the $24150 \mu \mathrm{m} \times 100 \mu \mathrm{m}$ pixel cell for the two detectors. In these 25 sensors the charge drifts along the thickness of the detector 26 allowing the charge cloud to diffuse across pixels. Figure 2 27 shows that significant charge sharing is observed at $20 \mu \mathrm{m}$ 28 where the charge collected by the pixel cell is lower than the 29 charge collected at the center. This effect is more evident in 30 Fig 3 where the charge collected at the corners of 4 pixel cells 31 is plotted.

32 Both mono and poly crystals show similar diffusion properties 33 with a cloud extending for 20-25 $\mu \mathrm{m}$ with a slightly greater 34 diffusion cloud in the poly-crystal. 35
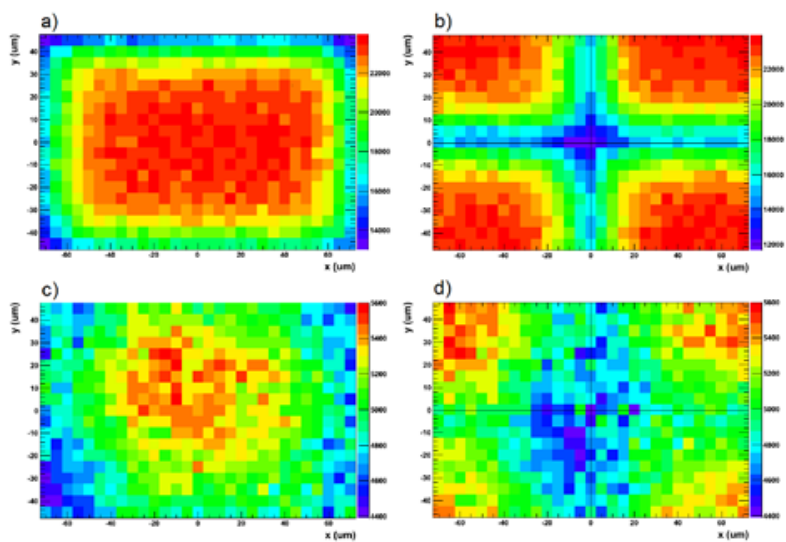

36

Figure 2: Plots a) and c) show the mean charge collected as a function of the 38 coordinates of the telescope predicted track impact point on a single pixel cell 39 for the mono and poly crystals respectively. Plots b) and d) show the mean 40 charge collected as a function of the coordinates of the track impact point 41 centered at the corner between four adjacent pixels for the mono and poly 42 crystals respectively.

\section{3}

\section{Efficiency}

45

46 Efficiency measurements are strongly biased by the fact that

47 the amount of charge collected in the poly-crystal is just above 48 the minimum threshold of about $2400 e^{-}$that we could apply to
49 the ROC. Figure 3 shows the almost perfect efficiency for the 50 mono-crystal that reaches $99.8 \%$ and, on the other hand, the 51 low efficiency, due to threshold settings, of the poly-crystal 52 that is only around $65 \%$. The two types of data points refer to 53 the sole efficiency of the pixel pointed by the track (green 54 points) and the combined efficiency of the two adjacent pixels 55 (blue points). While the combined efficiency, for the mono56 crystal, is constant and does not show any appreciable 57 degradation even in the region between pixels, for the poly58 crystal instead there is a further decrease in efficiency due to 59 the fact that the charge is split between two pixels thus 60 increasing the probability to be under threshold.

61
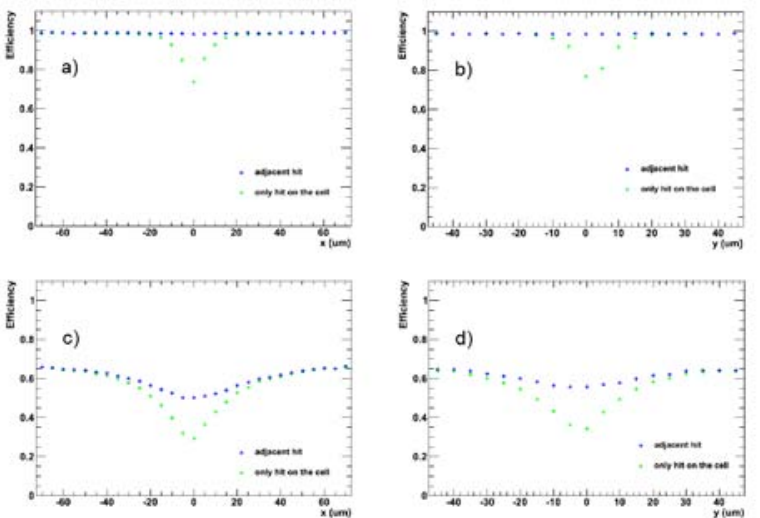

Figure 3: The green points show the detection efficiency of the sole pixel 64 pointed by the track as a function of the distance of the track impact point from 65 the boundary of the two adjacent pixels while the blue points show the combined 66 efficiency of the pixel pointed by the track and the adjacent one on the same 67 column, histograms a) for the mono-crystal, and c) for the poly-crystal, or on 68 the same row, histograms b) for the mono-crystal and d) for the poly-crystal.

69

\section{Conclusions}

71

72 Preliminary results on two different, un-irradiated diamond 73 sensors tested extensively in a beam-test at Fermilab have 74 been shown in this paper. The purity of the mono-crystal 75 detector allows for a much higher charge collection distance 76 that results in the collection of a much higher quantity of 77 charge and thus showing very high detection efficiency $78(99.8 \%)$. On the other hand, instead, the impurities in the poly79 crystalline structure of the other diamond detector limit the 80 amount of charge collected and thus results in poor 81 efficiencies (65\%) since the ROC cannot be set at a threshold 82 far away from the Landau peak of this type of detector. Both 83 diamonds show similar charge diffusion properties though. 84

85 Acknowledgments: The authors would like to express 86 gratitude to the Fermilab test-beam facility personnel, and in 87 particular to Aria Soha, for their support during all test-beam 88 activities.

89

\section{Bibliography}

91 [1] "The CMS Tracker Technical Design Report", CMS/LHC 98-6.

92 [2] “A Telescope Using CMS PSI46 Pixels and the CAPTAN for

93 Acquisition and Control over Gigabit Ethernet", R. A. Rivera,

94 M. Turqueti, L. Uplegger, 2009 IEEE Proc. Nuclear Science

95 Symposium.

96[3] "Design and Performance of the CMS Pixel Detector Readout 97 Chip", H.C. Kästli et al, arXiv:physics/0511166.

98[4] "CAPTAN: A hardware architecture for integrated dato 99 acquisition, control, and analysis for detector development", $\mathrm{M}$. 100 Turqueti et al, FERMILAB-PUB-08-527-CD 